\section{Relação entre oferta de diagnóstico e tratamento da sífilis na atenção básica sobre $a$ incidência de sífilis gestacional e congênita}

\author{
Relationship between the supply of syphilis \\ diagnosis and treatment in primary care and \\ incidence of gestational and congenital syphilis
}

\author{
Relación entre ofrecer un diagnóstico y el \\ tratamiento de la sífilis dentro de la atención \\ básica, respecto a la incidencia de sífilis \\ gestacional y congénita
}

\section{Resumo}

O Brasil tem registrado aumento nas incidências de sífilis gestacional e congênita, revelando-se como um importante problema de saúde pública no país. $O$ trabalho teve como objetivo analisar a relação entre as ofertas de diagnóstico e tratamento da sífilis na atenção básica e as incidências de sífilis gestacional e congênita. Foi realizado estudo ecológico analisando as incidências desses agravos e a cobertura de ações diagnósticas e terapêuticas na atenção básica. A amostra do estudo foi composta por municípios com população acima de 20.000 habitantes, com cobertura da atenção básica superior a 50\% e nos quais a maioria das equipes foi avaliada no segundo ciclo do Programa de Melhoria do Acesso e da Qualidade na Atenção Básica. Para analisar a efetividade das ações de detecção e tratamento foi desenvolvido o Índice de Variação da Transmissão Vertical de Sífilis. A administração da penicilina e a realização de teste rápido nesses municípios obtiveram medianas iguais a 41,9\% e 67,14\%, respectivamente, com diferenças regionais. A mediana da incidência de sífilis gestacional foi 6,24 (IIQ: 2,63-10,99) em municípios com maior oferta de teste rápido, e de 3,82 (IIQ: 0,00-8,21) naqueles com oferta inferior, apontando aumento na capacidade de detecção. Municípios com redução da transmissão vertical apresentavam maiores medianas dos percentuais de equipes com oferta dos testes rápidos (83,33\%; IIQ: 50,00-100,00) e realização de penicilina (50,00\%; IIQ: 11,10-87,50), demonstrando relação entre estas ações e a redução de sífilis congênita. Os achados indicam a necessidade de ampliação dessas ofertas e reforça a importância na redução da transmissão vertical.

Sífilis Congênita; Saúde Materno-infantil; Atenção Primária à Saúde;

Incidência; Avaliação em Saúde
Daniela Cristina Moreira Marculino de Figueiredo 1,2 Alexandre Medeiros de Figueiredo 1

Tanise Kely Bezerra de Souza 1,2

Graziela Tavares 3

Rodrigo Pinheiro de Toledo Vianna ${ }^{1,2}$

doi: 10.1590/0102-311X00074519

Correspondência

D. C. M. M. Figueiredo

Universidade Federal da Paraíba.

Departamento de Estatística, Conjunto Presidente Castelo

Branco III, João Pessoa, PB 58033-455, Brasil.

dla.moreira@yahoo.com.br

1 Universidade Federal da Paraíba, João Pessoa, Brasil.

2 Programa de Pós-graduação em Modelos de Decisão e Saúde, Fundação Oswaldo Cruz, Brasília, Brasil.

3 Programa de Pós-graduação em Políticas Públicas em Saúde Fundação Oswaldo Cruz, Brasília, Brasil. 


\section{Introdução}

Países e territórios das Américas estabeleceram em 2010 planos de ação para a eliminação da transmissão vertical de HIV e sífilis congênita, definindo como meta uma taxa de incidência de sífilis congênita de $\leq$ 0,5 caso por mil nascidos vivos, até o ano de 2015 1. Em 2014, 17 desses países e territórios relataram dados indicando a eliminação da transmissão de mãe para filho tanto do HIV quanto da sífilis 1 .

No Brasil, observa-se aumento nas incidências de sífilis, sífilis gestacional e sífilis congênita 2. A taxa de incidência da sífilis congênita e as taxas de detecção de sífilis em gestante por mil nascidos vivos aumentaram de modo expressivo entre os anos de 2010 a 2017, passando de 2,4 para 8,6 e de 3,5 para 17,2 casos por mil nascidos vivos, respectivamente 2 , sendo também percebido o aumento nestas incidências em outros países do mundo 3,4,5.

O diagnóstico e tratamento da sífilis gestacional são realizados na atenção básica, que nos últimos anos vem apresentado um aumento no número de equipes, atingindo cobertura populacional de $87,17 \%$ em 2014 6. Esse aumento de cobertura está associado à melhoria de indicadores como a redução das internações por causas sensíveis e redução da mortalidade infantil 7,8,9.

Como estratégias de diagnóstico de sífilis gestacional na atenção básica existem a triagem por meio do Venereal Disease Research Laboratory Test (VDRL) e o teste rápido (treponêmico) no primeiro e terceiro trimestres de gestação no pré-natal e na ocasião da internação para o parto ou curetagem, e o tratamento com a penicilina G benzatina 10. Quando as gestantes apresentam resultado reagente, o controle do tratamento e da cura deve ser realizado usando-se o VDRL.

Apesar de avanços no Sistema Único de Saúde (SUS), o combate à sífilis congênita com base no tratamento da sífilis gestacional permanece como desafio, sobretudo ao observarmos o seu aumento ao longo dos anos e por considerarmos que a ocorrência da sífilis congênita indica fragilidades na atenção ao pré-natal, sendo, portanto, um evento sentinela para o monitoramento do acesso e da qualidade da atenção básica 11 .

Em 2011, o Brasil instituiu o Programa Nacional de Melhoria do Acesso e da Qualidade na Atenção Básica (PMAQ-AB), alinhado aos objetivos e desafios da Política Nacional de Atenção Básica (PNAB), buscando produzir padrão de qualidade comparável nacional, regional e localmente para auxiliar no fortalecimento das suas ações. Dentre as etapas/fases do PMAQ-AB há a avaliação externa das equipes de atenção básica/saúde da família, que no Módulo II apresenta questões diretamente relacionadas ao diagnóstico e tratamento da sífilis realizados pelas equipes de atenção básica 12. A avaliação externa do segundo ciclo do PMAQ-AB contou com a adesão voluntária de 5.070 (91\%) municípios e 30.424 (90\%) equipes de atenção básica, que foram avaliadas entre 2013 e o primeiro semestre de 2014.

O presente trabalho tem como objetivo analisar a relação entre as ofertas de diagnóstico e tratamento para sífilis na atenção básica e as incidências municipais de sífilis gestacional e congênita. Busca-se também avaliar o impacto dessas ações na redução da transmissão vertical, por meio de indicador que mensure essas variações.

\section{Métodos}

Trata-se de estudo ecológico, em que as variáveis independentes foram o percentual de equipes da atenção básica que realizavam ações de diagnóstico e tratamento para sífilis no ano de 2014, e as variáveis dependentes foram as incidências municipais de sífilis gestacional e congênita no mesmo ano.

As incidências, relativas ao ano de 2014, foram calculadas considerando-se o número de casos notificados no Sistema de Informação de Agravos de Notificação (SINAN), por município de residência, e os dados sobre nascidos vivos obtidos do Sistema de Informações sobre Nascidos Vivos (SINASC), por local de residência da mãe, ambos disponíveis na página de Internet do Departamento de Informática do SUS (DATASUS; http://datasus.saude.gov.br/). Quanto às ações de diagnóstico e tratamento de sífilis gestacional nas unidades básicas de saúde (UBS), os dados foram coletados nas bases do Módulo II do PMAQ-AB, em sítio eletrônico do Ministério da Saúde, de acesso livre (http:// dab.saude.gov.br/portaldab/ape_pmaq.php?conteudo=microdados). 
Para a análise das variáveis selecionadas, observaram-se as seguintes ações: realização de penicilina G benzatina, realização de VDRL e teste rápido de sífilis na UBS. Para cada município foi calculado o percentual de equipes que realizavam tais ações conforme a fórmula:

Percentual de equipes com ação $X=\frac{\text { Equipes avaliadas no } P M A Q-A B \text { que realizaram a ação } X \text { no município }}{\text { Total de equipes avaliadas no PMAQ-AB no município }}$

Em seguida, foram criadas variáveis categóricas com base nesses percentuais, dividindo os municípios em um subgrupo no qual mais da metade das equipes do município realizavam a ação analisada e outro subgrupo com valores inferiores a este ponto de corte.

Consideraram-se como critérios de elegibilidade todos os municípios com população acima de 20 mil habitantes, onde no mínimo a metade das suas equipes foi avaliada no PMAQ-AB e a cobertura populacional estimada na atenção básica superior a 50\%, conforme metodologia de cálculo e dados do Departamento da Atenção Básica (DAB) do Ministério da Saúde (Figura 1).

Realizou-se análise descritiva para considerar variações regionais da oferta dessas ações na atenção básica do país. Os dados de incidência não preencheram os critérios de normalidade e, portanto, foram empregados métodos estatísticos não paramétricos. Foi realizada análise bivariada usando-se

\section{Figura 1}

Caracterização da população e amostra do estudo com base nos critérios de elegibilidade. Brasil, 2014.

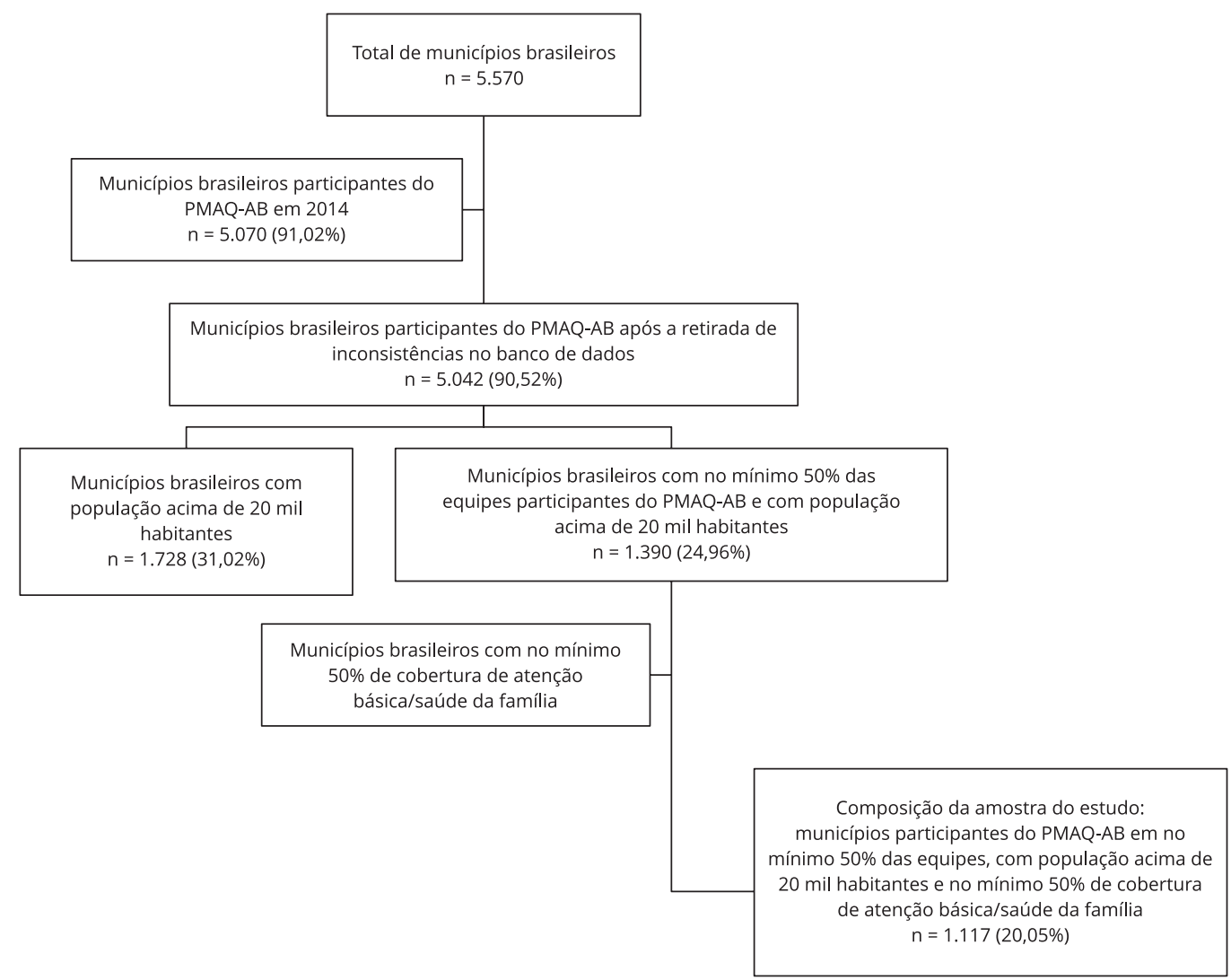

PMAQ-AB: Programa Nacional de Melhoria do Acesso e da Qualidade na Atenção Básica. 
o teste de Mann-Whitney para comparar a oferta de teste rápido para sífilis e o tratamento com penicilina na maioria das equipes e as incidências de sífilis gestacional (IncSG) e congênita (IncSC).

Para uma análise adicional, foi elaborado um indicador visando a mensurar a efetividade das atividades de diagnóstico e tratamento de sífilis durante o pré-natal, sendo este denominado como Índice de Variação da Transmissão Vertical de Sífilis (IVTVS). Partiu-se do pressuposto de que ações de pré-natal efetivas seriam capazes de identificar e tratar casos de sífilis gestacional, evitando a transmissão vertical e reduzindo a incidência de sífilis congênita. O referido indicador foi calculado segundo a fórmula:

$$
I V T V S=I n c S C-I n c S G
$$

Valores positivos do IVTVS indicam que a incidência de sífilis congênita foi superior à incidência de sífilis gestacional, sugerindo que parte ou a totalidade dos casos de sífilis não foi identificada e/ou tratada adequadamente durante o pré-natal. Os valores negativos do IVTVS indicam que parte e/ou a totalidade dos casos de sífilis foi tratada adequadamente e reduziu a transmissão vertical. Valores iguais a zero representam municípios sem incidência de sífilis gestacional e congênita no período ou situações nas quais as incidências de sífilis congênita e gestacional foram iguais, demonstrando fragilidades na assistência.

Por fim, os municípios foram categorizados baseando-se no IVTVS e foi realizada análise bivariada por meio do teste de Kruskal-Wallis, para avaliar a relação entre a capacidade de redução da transmissão vertical de sífilis e os percentuais de equipes da atenção básica do município que realizavam penicilina e/ou teste rápido para sífilis.

Em atenção às recomendações contidas na Resolução no 466/2012, do Conselho Nacional de Saúde, o projeto deste estudo foi submetido e aprovado pelo Comitê de Ética em Pesquisa com Seres Humanos, da Universidade Federal da Paraíba - Centro de Ciências Médicas (CEP/CCM/UFPB), sob CAAE no 98713118.7.0000.8069 e parecer no 2.930.476, em 27 de setembro de 2018. Os autores declararam não haver conflito de interesses.

\section{Resultados}

A avaliação do PMAQ-AB em 2014 foi realizada em 5.070 (91,02\%) municípios brasileiros, destes, 1.117 (20,05\%) preencheram os critérios de elegibilidade e compuseram a amostra para o presente trabalho (Figura 1). Desses, 93 (8,33\%) estavam na Região Norte, 493 (44,14\%) no Nordeste, 95 (8,50\%) no Centro-oeste e, respectivamente, 295 (26,41\%) e 141 (12,62\%) nas regiões Sudeste e Sul (Tabela 1)

Em 750 (67,14\%) municípios no Brasil, a maioria das equipes afirmou que realizava teste rápido para sífilis, sendo a menor distribuição identificada na Região Sudeste ( $\mathrm{n}=167 ; 56,61 \%)$ e a maior na Região Norte ( $\mathrm{n}=78 ; 83,87 \%)$. Quanto ao VDRL no município, observamos que 1.109 (99,28\%) referiram afirmativamente quanto à realização, com todas as regiões apresentando frequências superiores a 95\%. (Tabela 1). Em 468 (41,9\%) dos municípios brasileiros, mais de 50\% das equipes de atenção básica/saúde da família referiram administrar a penicilina G benzatina, variando de $68(73,12 \%)$ na Região Norte a 67 (22,71\%) no Sudeste.

Ao comparar a realização do teste rápido para sífilis e o tratamento com penicilina G benzatina com a incidência de sífilis gestacional, observou-se que as medianas da incidência de sífilis gestacional foram maiores no grupo de municípios que ofertaram penicilina e teste rápido na maioria das equipes, respectivamente, 6,50 e 6,24 casos para mil nascidos vivos, do que no grupo com menor oferta destas ações, 4,50 e 3,82 casos para mil nascidos vivos (Tabela 2).

Quanto ao uso da penicilina e a incidência de sífilis congênita, a mediana foi de 2,67 casos para mil nascidos vivos no grupo de municípios com maior oferta do tratamento nas UBS, e de 2,80 casos para mil nascidos vivos nos municípios com menor oferta (Tabela 2). Sobre a realização de teste rápido, a mediana foi de 2,86 casos para cada mil nascidos vivos nos municípios onde mais de $50 \%$ das equipes realizavam este procedimento, e 2,50 casos para cada mil nascidos vivos nos municípios com oferta do teste rápido em menos da metade das equipes avaliadas (Tabela 2). 


\section{Tabela 1}

Descrição da amostra considerando quantitativo e proporção de municípios em que mais da metade das equipes, avaliadas no Programa Nacional de Melhoria do Acesso e da Qualidade na Atenção Básica (PMAQ-AB), realizavam diagnóstico (oferta de teste rápido e Venereal Disease Research Laboratory Test - VDRL) e tratamento (administração de penicilina). Brasil, 2014.

\begin{tabular}{|c|c|c|c|c|c|c|c|c|c|}
\hline \multirow[t]{2}{*}{ Região } & \multicolumn{2}{|c|}{$\begin{array}{l}\text { Municípios com } \\
\text { administração de } \\
\text { penicilina }\end{array}$} & \multicolumn{2}{|c|}{$\begin{array}{l}\text { Municípios com } \\
\text { realização de teste } \\
\text { rápido para sífilis }\end{array}$} & \multicolumn{2}{|c|}{$\begin{array}{l}\text { Municípios com } \\
\text { realização de VDRL }\end{array}$} & \multicolumn{2}{|c|}{$\begin{array}{l}\text { Municípios } \\
\text { avaliados * }\end{array}$} & \multirow{2}{*}{$\begin{array}{l}\text { Cobertura da } \\
\text { atenção primária } \\
\text { à saúde nos } \\
\text { municípios } \\
\text { Mediana (\%) }\end{array}$} \\
\hline & $\mathbf{n}$ & $\%$ & $\mathbf{n}$ & $\%$ & $\mathbf{n}$ & $\%$ & $\mathbf{n}$ & $\%$ & \\
\hline Norte & 68 & 73,12 & 78 & 83,87 & 89 & 95,70 & 93 & 8,30 & 86,70 \\
\hline Nordeste & 201 & 40,77 & 335 & 67,95 & 491 & 99,59 & 493 & 44,10 & 100,00 \\
\hline Centro-oeste & 59 & 62,11 & 66 & 69,47 & 93 & 97,89 & 95 & 8,50 & 86,70 \\
\hline Sudeste & 67 & 22,71 & 167 & 56,61 & 295 & 100,00 & 295 & 26,40 & 81,50 \\
\hline Sul & 73 & 51,77 & 104 & 73,76 & 141 & 100,00 & 141 & 12,60 & 78,80 \\
\hline Brasil & 468 & 41,90 & 750 & 67,10 & 1.109 & 99,30 & 1.117 & 100,00 & 91,70 \\
\hline
\end{tabular}

* Do total de 5.570 municípios brasileiros, 5.042 (90,52\%) participaram do PMAQ-AB, sendo que 1.117 (20,05\%) preencheram os critérios de elegibilidade para o estudo.

\section{Tabela 2}

Comparação da incidência de sífilis gestacional (InCSG) e congênita (InCSC) entre grupos de municípios com diferentes percentuais de equipes que realizam teste rápido e tratamento (penicilina benzatina). Brasil, 2014.

\begin{tabular}{|c|c|c|c|c|c|c|}
\hline Variável & Mediana da IncSG & IIQ & Valor de $p$ & Mediana da IncSC & IIQ & Valor de $p$ \\
\hline Realização de penicilina benzatina & & & 0,001 * & & & 0,611 \\
\hline Em menos de $50 \%$ das equipes & 4,50 & $1,97-9,44$ & & 2,80 & $0,00-6,88$ & \\
\hline Em mais de $50 \%$ das equipes & 6,50 & $2,34-11,48$ & & 2,67 & $0,00-6,33$ & \\
\hline Realização de teste rápido & & & $<0,001$ * & & & $0,016 * *$ \\
\hline Em menos de $50 \%$ das equipes & 3,82 & $0,00-8,21$ & & 2,50 & $0,00-5,93$ & \\
\hline Em mais de $50 \%$ das equipes & 6,24 & $2,63-10,99$ & & 2,86 & $0,00-7,00$ & \\
\hline
\end{tabular}

IIQ: intervalo interquartílico.

Nota: valor de $p$ de Mann-Whitney: * $p<0,001$; ** $p<0,05$.

A análise do IVTVS demonstrou que, na maioria dos municípios brasileiros (59\%), a incidência de sífilis congênita foi inferior a de sífilis gestacional. Em 245 (21,9\%) municípios, essas incidências foram iguais e em $213(19,1 \%)$ a incidência de sífilis congênita foi maior do que a incidência de sífilis gestacional (Tabela 3). A Região Centro-oeste apresentou o maior percentual de municípios que registraram incidência de sífilis congênita inferior a de sífilis gestacional $(83,2 \%)$ e a Região Nordeste o menor percentual (48,3\%).

$\mathrm{Na}$ análise bivariada, os municípios com IVTVS negativo apresentaram maiores percentuais de realização de teste rápido e penicilina com medianas, respectivamente, de 83,3\% e 50\% (Tabela 4). Os municípios com IVTVS positivo e igual a zero registraram mediana do percentual de equipes que ofertaram teste rápido, respectivamente, de 70,8\% e 62,5\% e de realização de penicilina de $25 \%$ e 33,3\% (Tabela 4). A comparação dos percentuais dessas ações usando-se o teste de Kruskal-Wallis verificou que as variáveis percentual de equipes que realizam teste rápido ( $<0,001)$ e percentual de equipes que realizam penicilina $(\mathrm{p}=0,001)$ apresentaram diferenças significativas entre o grupo com IVTVS negativo e os outros dois grupos. Os grupos com IVTVS positivo e zero não diferiram entre si $(\mathrm{p}>0,05)$. 


\section{Tabela 3}

Classificação dos municípios da amostra com base no Índice de Variação da Transmissão Vertical de Sífilis (IVTVS) considerando as macrorregiões do Brasil, 2014

\begin{tabular}{|c|c|c|c|c|c|c|c|}
\hline \multirow[t]{2}{*}{ Categoria } & \multicolumn{2}{|c|}{$\begin{array}{l}\text { Municípios com IncSC maior } \\
\text { que IncSG (IVTVS positivo) }\end{array}$} & \multicolumn{2}{|c|}{$\begin{array}{l}\text { Municípios com IncSC igual à } \\
\text { IncSG (IVTVS }=0 \text { ) }\end{array}$} & \multicolumn{2}{|c|}{$\begin{array}{l}\text { Municípios com IncSC menor } \\
\text { que IncSG (IVTVS negativo) }\end{array}$} & \multirow{2}{*}{$\begin{array}{c}\text { Total } \\
\mathbf{n}\end{array}$} \\
\hline & $\mathbf{n}$ & $\%$ & $\mathbf{n}$ & $\%$ & $\mathbf{n}$ & $\%$ & \\
\hline Norte & 12 & 12,90 & 16 & 17,20 & 65 & 69,90 & 93 \\
\hline Nordeste & 139 & 28,20 & 116 & 23,53 & 238 & 48,27 & 493 \\
\hline Centro-oeste & 4 & 4,21 & 12 & 12,63 & 79 & 83,16 & 95 \\
\hline Sudeste & 51 & 17,30 & 70 & 23,72 & 174 & 58,98 & 295 \\
\hline Sul & 7 & 4,97 & 31 & 21,98 & 103 & 73,05 & 141 \\
\hline Brasil & 213 & 19,07 & 245 & 21,93 & 659 & 59,00 & 1.117 \\
\hline
\end{tabular}

IncSC: incidência de sífilis congênita; IncSG: incidência de sífilis gestacional.

\section{Tabela 4}

Comparação do percentual de equipes que realizavam penicilina benzantina e teste rápido para sífilis entre grupos de municípios categorizados pelo Índice de Variação da Transmissão Vertical de Sífilis (IVTVS). Brasil, 2014.

\begin{tabular}{lcccc}
\hline Grupo de municípios por IVTVS & $\begin{array}{c}\text { Teste rápido } \\
\text { (Mediana - \%) }\end{array}$ & IIQ & Valor de p & $\begin{array}{c}\text { Penicilina } \\
\text { (Mediana - \%) }\end{array}$ \\
\hline IncSC > IncSG (IVTVS positivo) & $70,83 *$ & $33,30 \%-96,30 \%$ & $25,00 * *$ \\
IncSC = IncSG (IVTVS = 0) & $62,50 *$ & $33,30 \%-95,60 \%$ & $<0,001 * * *$ & $33,30 * *$ \\
IncSC < IncSG (IVTVS negativo) & $83,33 * *$ & $50,00 \%-100,00 \%$ & $0,00 \%-80,90 \%$ & $11,10 \%-87,50 \%$ \\
\hline
\end{tabular}

IIQ: intervalo interquartílico; IncSC: incidência de sífilis congênita; IncSG: incidência de sífilis gestacional.

* Equipes que realizavam teste rápido na unidade de saúde;

** Equipes que realizavam a administração de penicilina na unidade de saúde.

Nota: valor de $p$ de Kruskal-Wallis: *** $p<0,001 ; \# p=0,001$.

\section{Discussão}

O estudo evidenciou que a realização de ações de diagnóstico para sífilis (testes treponêmicos e/ ou não treponêmicos) estavam disponíveis em mais de 95\% das equipes dos municípios avaliados, atingindo a meta proposta no plano de ação para a eliminação da transmissão vertical de HIV e sífilis congênita da Organização Pan-Americana da Saúde (OPAS) 1. Em 2014, a triagem para sífilis em gestantes, com no mínimo uma consulta de pré-natal, aumentou em 3\% nos países das Américas e Caribe entre 2011 e 2014, chegando a 80\% em 2014, com ampliação também dos países que usaram testes rápidos para identificar gestantes com sífilis 1. No Brasil, a disponibilização de teste rápido passou de 31.500 em 2011 para 3.156.410 em 2014, mas com distribuições diferentes e inferiores em relação à quantidade e estimativa de gestantes nos estados 13. Assim como em outros agravos, o aumento da capacidade de identificação de pessoas portadoras assintomáticas de sífilis resultou em aumento de casos de sífilis adquirida, gestacional e congênita. Equipes que realizavam teste rápido ampliaram de forma significativa a identificação e notificação de casos em gestantes, possibilitando o cuidado oportuno no pré-natal. Nos municípios avaliados, entretanto, cerca de $1 / 3$ das equipes não ofertava teste rápido apontando que ainda seria necessária a ampliação desta medida.

Os dados do estudo revelam que havia limitações no acesso ao tratamento para sífilis na atenção básica, e uma maior variabilidade regional. Nas regiões Sudeste e Nordeste do país, menos de 50\% 
dos municípios estudados realizavam a aplicação de penicilina em mais da metade de suas equipes, reduzindo o acesso ao tratamento e perpetuando a cadeia de transmissão.

A mediana das incidências de sífilis gestacional dos municípios com maior oferta de teste rápido foi 1,63 vez o valor encontrado em municípios com menor oferta e aponta para uma maior capacidade de detecção. Por sua vez, a incidência de sífilis congênita foi 1,14 vez maior nesses municípios, revelando que o aumento na capacidade diagnóstica não foi acompanhado de uma capacidade proporcional de tratamento em tempo oportuno e uma maior redução na incidência de sífilis congênita.

A mediana das incidências de sífilis gestacional, nos municípios nos quais a maioria das UBS realizava penicilina benzatina, foi superior aos municípios com menor acesso ao tratamento na atenção básica, e a incidência de sífilis congênita foi inferior. Dessa forma, evidencia-se que a oferta de realização de penicilina na atenção básica está relacionada a uma redução na transmissão vertical de sífilis e deve ser ampliada.

O percentual de municípios da amostra com redução da transmissão vertical de sífilis (IVTVS negativo) foi maior nas regiões Centro-oeste, Sul e Norte do país, sendo estas as três regiões nas quais o estudo evidenciou maior oferta de teste rápido e realização de penicilina benzatina na atenção básica. Esses achados estão de acordo com um trabalho anterior que aponta que essas foram as regiões com menores percentuais de transmissão vertical ${ }^{14}$. O grupo de municípios com maior redução da transmissão vertical apresentou uma maior mediana de realização de teste rápido e penicilina benzatina, o que corrobora a percepção de que o aumento do acesso à atenção básica é uma importante estratégia para o enfrentamento da epidemia de sífilis no país.

Apesar do avanço no acesso a ações de diagnóstico e tratamento na atenção básica, as incidências de sífilis congênita e gestacional ainda distam das metas preconizadas internacionalmente ${ }^{2}$. Os dados nacionais revelam que os esforços impetrados até aqui têm sido insuficientes para quebrar a cadeia de transmissão, o que resulta em uma tendência crescente nas incidências de sífilis adquirida, gestacional e congênita em todas as regiões do país 2 .

A ampliação do acesso ao pré-natal ocorrido no país nos últimos anos e a ampliação das ações de diagnóstico de sífilis não foram suficientes para reduzir os casos de sífilis congênita em função de barreiras importantes para a realização do diagnóstico e tratamento. Dados de uma pesquisa de abrangência nacional realizada entre 2011 e 2012, revelam que apenas $89,1 \%$ das gestantes realizaram pelo menos uma sorologia para sífilis no pré-natal 1 , sendo este valor inferior à meta de $95 \%$ de cobertura de testagem 14 .

Os dados do estudo demonstram que, mesmo em municípios com mais de 20 mil habitantes, parte significativa das equipes de saúde não realizava a administração de penicilina. Uma possível justificativa para esse resultado está na recusa da administração do medicamento pelo receio dos profissionais da atenção básica quanto à ocorrência de reações anafiláticas e à limitação em recursos disponíveis para atender os eventos de maior gravidade 15 , embora já seja comprovada a segurança em sua administração na atenção básica e os baixos índices de reações adversas, em que possíveis riscos não ultrapassam seus benefícios 16 .

Outra possibilidade para tal resultado foi o desabastecimento de penicilina no país e no mundo 17 . Em junho de 2014, o Ministério da Saúde registrou o desabastecimento da penicilina no Brasil 18, e junto aos estados e municípios buscou estratégias e soluções 19 , sobretudo em relação à penicilina benzatina e cristalina. Entendendo a relevância do medicamento para a prevenção da sífilis congênita, no mesmo ano foram iniciadas as tentativas de aquisição e distribuição da penicilina pelo Ministério da Saúde, por intermédio de negociação tripartite com estados e municípios, já que seria destes a responsabilidade pela compra do medicamento. Apesar da coleta de dados do segundo ciclo ter sido realizada em período anterior à notificação oficial do desabastecimento, é possível que os processos de aquisição e oferta de penicilina pelos municípios possam ter sido prejudicados e impactado nos resultados do PMAQ-AB. Segundo nota técnica emitida 18 , a distribuição da penicilina em aquisição emergencial só iniciou no primeiro semestre do ano de 2016, o que pode ter reduzido ainda mais o percentual de equipes que realizavam penicilina nos anos subsequentes ao estudo.

No período em que foram coletados os dados de incidência para sífilis gestacional e congênita, era considerado tratamento adequado na gestante quando realizado com penicilina benzatina, concluído 30 dias antes do parto, e tratamento adequado do parceiro, estando estas ações documentadas adequadamente 20,21. Atualmente, acompanhando as recomendações internacionais 22 , o tratamento 
do parceiro deixou de ser critério para avaliar a adequação do tratamento da gestante 23 , embora a estratégia de notificação e tratamento do parceiro seja recomendada tanto para prestar cuidados aos indivíduos expostos como para prevenir a reinfecção da gestante 24.

Estudos controlados exploraram a notificação do parceiro como componente da triagem e tratamento em gestantes com sífilis, e evidenciaram que o tratamento de parceiros estava relacionado à redução de desfechos adversos da gestação em comparação com os grupos em que eles não foram notificados. Porém, esses não investigaram a associação entre a notificação do parceiro e a diminuição da incidência de sífilis congênita 25,26,27. Considera-se que a notificação e tratamento dos parceiros seja uma estratégia global de redução da incidência geral de sífilis, atuando na redução da incidência de sífilis gestacional.

No Brasil, há evidências que apontam para o tratamento insuficiente dos parceiros de gestantes com diagnóstico de sífilis 28 . No país, os parceiros sexuais das gestantes notificadas com sífilis são comunicados pela própria gestante ou por um profissional de saúde 29 . Não há trabalhos suficientes que avaliem a efetividade da comunicação do diagnóstico aos parceiros em relação a aspectos cruciais como a importância do tratamento mesmo em casos assintomáticos, a possibilidade de ocorrer reinfecção, bem como das consequências quando o tratamento não for realizado corretamente 30 . Essa dificuldade de abordagem aos parceiros pode ser um dos elementos importantes para a baixa adesão e grande número de falhas terapêuticas nestes usuários. Na maioria dos países europeus 31 , as ações de notificação de parceiros de pessoas com diagnósticos de infecções sexualmente transmissíveis (ISTs) são de responsabilidade dos profissionais de saúde, o que poderia ser uma ação implantada no país.

Para o tratamento adequado, parte dos casos demanda consultas frequentes a serviços de saúde, o que pode representar custos com deslocamentos e faltas a atividades laborais. Assim, além da acessibilidade geográfica é preciso considerar aspectos relacionados à acessibilidade funcional, como horário de funcionamento da UBS, e organização do fluxo de atendimento destes usuários para que os mesmos se sintam acolhidos. Os estudos também evidenciam que há uma maior incidência de sífilis em gestantes em maior situação de vulnerabilidade social, o que pode ampliar a dificuldade de acesso 14,21.

Nos Estados Unidos, os casos de sífilis congênita estão associados ao início tardio ou ausência de pré-natal, ocorrendo, sobretudo, nas gestantes negras e hispânicas no sul do país 32,33. Um trabalho realizado com 23 gestantes americanas revelou exemplos como a instabilidade de moradia (mulheres que viviam em abrigos, moravam com os pais ou haviam sido despejadas recentemente), parceiros privados de liberdade ou as gestantes tinham vivenciado período de encarceramento 34 . Assim, percebe-se que a pobreza, o isolamento social, a instabilidade no controle da vida pessoal e profissional, bem como questões relacionadas à moradia, alimentação, educação, entre outros, são condições que estão relacionadas ao aumento do risco de sífilis congênita 35 .

No Brasil, estudos regionais revelam relação entre a sífilis congênita e a baixa escolaridade da mãe $36,37,38,39$, não inserção no mercado de trabalho formal, uso de drogas ilícitas, estratos sociais mais desfavorecidos 40,41, raça/cor parda ou negra 42,43 e a idade inferior a 30 anos $28,42,43$.

Trabalhos realizados em países latino-americanos 44,45,46,47 identificaram um número proporcionalmente importante de gestantes indígenas com diagnóstico de sífilis no Brasil, Peru e Bolívia, reforçando a percepção de dificuldade de acesso destas mulheres ao pré-natal, diagnóstico e tratamento para a sífilis, considerando, ainda, que há lacunas relacionadas à notificação de sífilis congênita e gestacional nesta população 1 .

A sífilis é uma doença sexualmente transmissível, por isto, seu diagnóstico na gestação desperta tensões tanto por suas repercussões biológicas, como o risco de malformação no concepto, quanto por suas repercussões sociais. Sentimentos de ansiedade e tristeza associados à notícia de possíveis problemas no concepto constituem um potencializador das vulnerabilidades existentes, dificultando a adesão ao tratamento e acompanhamento ${ }^{29}$. No âmbito social, o diagnóstico desperta sentimentos de raiva, culpa, insegurança, receio de comunicar ao parceiro e medo de ruptura do relacionamento 30. Portanto, o enfrentamento da epidemia de sífilis demanda habilidades de comunicação, ações intersetoriais, ações de vigilância em saúde, postura ética e garantia de confidencialidade das informações por parte de toda a equipe profissional. Recentemente, uma agenda de ações estratégicas 19 para a redução da sífilis adquirida, gestacional e congênita no Brasil foi estabelecida pelos gestores do SUS. Essa iniciativa contempla a ampliação do diagnóstico por meio do teste rápido, do número de equipes que realizam penicilina na atenção básica, integração das redes de atenção e das esferas governamen- 
tais, e fortalecimento das ações de prevenção e tratamento dos parceiros sexuais das gestantes. Essas intervenções estão em consonância com os achados deste estudo e seu impacto deve ser avaliado em pesquisas futuras.

O delineamento deste trabalho não nos permite afirmar que há relação de causalidade entre a expansão do acesso a métodos diagnósticos e terapêuticos na atenção básica e a redução da transmissão vertical, entretanto, os dados sugerem associação entre a ampliação do acesso e redução da transmissão vertical no grupo de municípios avaliados. As políticas implantadas precisam ser continuadas para que os objetivos traçados para longo prazo sejam alcançados. Assim, é importante prosseguir no processo de monitoramento e realizar estudos adicionais, utilizando outras metodologias, especialmente para identificar pontos de ajustes preferencialmente no tocante ao desenvolvimento de tecnologias leves e leve-duras que possibilitem melhor adesão ao tratamento.

\section{Colaboradores}

D. C. M. M. Figueiredo, A. M. Figueiredo e R. P. T. Vianna participaram da concepção do estudo, desenho do projeto, organização e análise dos dados, redação e revisão crítica do conteúdo do artigo. T. K. B. Souza participou da organização e análise dos dados e revisão do conteúdo do artigo. G. Tavares colaborou na redação e revisão do artigo. Todos os autores aprovaram a versão final a ser publicada.

\section{Informações adicionais}

ORCID: Daniela Cristina Moreira Marculino de Figueiredo (0000-0001-9443-1191); Alexandre Medeiros de Figueiredo (0000-0003-1433-088X); Tanise Kely Bezerra de Souza (0000-0001-54196203); Graziela Tavares (0000-0003-4264-8879); Rodrigo Pinheiro de Toledo Vianna (0000-00025358-1967).

\section{Agradecimentos}

Ao Departamento de Atenção Básica do Ministério da Saúde.

\section{Referências}

1. Pan American Health Organization. Elimination of mother-to-child transmission of HIV and syphilis in the Americas (Update). Washington DC: Pan American Health Organization; 2015.

2. Secretaria de Vigilância em Saúde, Ministério da Saúde. Sífilis 2018. Boletim Epidemiológico 2018; 49(45).

3. Bowen V, Su J, Torrone E, Kidd D, Weinstock $\mathrm{H}$. Increase in incidence of congenital syphilis - United States, 2012-2014. MMWR Morb Mortal Wkly Rep 2015; 64:1241-5.

4. Choudhri Y, Miller J, Sandhu J, Leon A, Aho J. Infectious and congenital syphilis in Canada, 2010-2015. Can Commun Dis Rep 2018; 44:43-8.

5. Takahashi T, Arima Y, Yamagishi Y, Nishiki S, Kanai M, Ishikane M, et al. Rapid increase in reports of syphilis associated with men who have sex with women and women who have sex with men, Japan, 2012 to 2016. Sex Transm Dis 2018; 45:139-43

6. Departamento de Atenção Básica, Secretaria de Atenção à Saúde, Ministério da Saúde. Sistemas da atenção básica: histórico de cobertura. Brasília: Ministério da Saúde; 2014.

7. Carvalho SC, Mota E, Dourado I, Aquino R, Teles C, Medina MG. Hospitalizations of children due to primary health care sensitive conditions in Pernambuco State, Northeast Brazil. Cad Saúde Pública 2015; 31:744-54.

8. Rasella D, Harhay MO, Pamponet ML, Aquino $\mathrm{R}$, Barreto ML. Impact of primary health care on mortality from heart and cerebrovascular diseases in Brazil: a nationwide analysis of longitudinal data. BMJ 2014; 349:g4014.

9. Ceccon R, Bueno LMA, Hesler LZ, Kirsten KS, Portes VM, Viecili PRN. Mortalidade infantil e saúde da família nas unidades da federação brasileira, 1998-2008. Cad Saúde Colet (Rio J.) 2014; 22:177-83.

10. Phiske MM. Current trends in congenital syphilis. Indian J Sex Transm Dis 2014; 35:1220. 
11. Domingues RMSM, Saraceni V, Hartz ZMA, Leal MC. Sífilis congênita: evento sentinela da qualidade da assistência pré-natal. Rev Saúde Pública 2013; 47:147-57.

12. Departamento de Atenção Básica, Secretaria de Atenção à Saúde, Ministério da Saúde. Manual instrutivo do Programa Nacional de Melhoria do Acesso e da Qualidade da Atenção Básica (PMAQ). Brasília: Ministério da Saúde; 2013.

13. Departamento de Vigilância, Prevenção e Controle das IST, do HIV/AIDS e das Hepatites Virais, Secretaria de Vigilância em Saúde, Ministério da Saúde. Distribuição de testes rápidos de sífilis, por Unidade da Federação. Brasil, 2011 a 2017. Brasília: Ministério da Saúde; 2018.

14. Domingues RMSM, Leal MC. Incidência de sífilis congênita e fatores associados à transmissão vertical da sífilis: dados do estudo Nascer no Brasil. Cad Saúde Pública 2016; 32:e00082415.

15. Araujo MAL, Barros VL, Moura HJ, Rocha AFB, Guanabara MAO. Prevenção da sífilis congênita em Fortaleza, Ceará: uma avaliação de estrutura e processo. Cad Saúde Colet (Rio J.) 2014; 22:300-6.

16. Galvão TF, Silva MT, Serruya SJ, Newman LM, Klausner JD, Pereira MG, et al. Safety of benzathine penicillin for preventing congenital syphilis: a systematic review. PLoS One 2013; 8:e56463.

17. Nurse-Findlay S, Taylor MM, Savage M, Mello MB, Saliyou S, Lavayen M, et al. Shortages of benzathine penicillin for prevention of mother-to-child transmission of syphilis: an evaluation from multi-country surveys and stakeholder interviews. PLoS Med 2017; 14:e1002473.

18. Departamento de Vigilância, Prevenção e Controle das IST, do HIV/AIDS e das Hepatites Virais, Secretaria de Vigilância em Saúde, Ministério da Saúde. Nota Informativa no 006/2016. Brasília: Ministério da Saúde; 2016.

19. Departamento de Vigilância, Prevenção e Controle das IST, do HIV/AIDS e das Hepatites Virais, Secretaria de Vigilância em Saúde, Ministério da Saúde. Agenda de ações estratégicas para redução da sífilis no Brasil. Brasília: Ministério da Saúde; 2017.

20. Departamento de DST, Aids e Hepatites Virais, Secretaria de Vigilância em Saúde, Ministério da Saúde. Protocolo clínico e diretrizes terapêuticas para atenção integral às pessoas com infecções sexualmente transmissíveis. Brasília: Ministério da Saúde; 2015.

21. Magalhães DMS, Kawaguchi IAL, Dias A, Calderon IMP. Sífilis materna e congênita: ainda um desafio. Cad Saúde Pública 2013; 29:110920.

22. Centers for Disease Control and Prevention. Syphilis - CDC Fact Sheet Syphilis (Detailed). Atlanta: Centers for Disease Control and Prevention; 2017.
23. Departamento de DST, Aids e Hepatites Virais, Secretaria de Vigilância em Saúde, Ministério da Saúde. Protocolo clínico e diretrizes terapêuticas para atenção integral às pessoas com infecções sexualmente transmissíveis. Brasília: Ministério da Saúde; 2019.

24. Plotzker RE, Murphy RD, Stoltey JE. Congenital syphilis prevention: strategies, evidence, and future directions. Sex Transm Dis 2018; 45(9S Suppl 1):S29-37.

25. Bique Osman N, Challis K, Folgosa E, Cotiro $\mathrm{M}$, Bergström S. An intervention study to reduce adverse pregnancy outcomes as a result of syphilis in Mozambique. Sex Transm Infect 2000; 76:203-7.

26. Hira SK, Bhat GJ, Chikamata DM, Nkowane B, Tembo G, Perine PL, et al. Syphilis intervention in pregnancy: Zambian demonstration project. Genitourin Med 1990; 66:159-64.

27. Munkhuu B, Liabsuetrakul T, Chongsuvivatwong V, McNeil E, Janchiv R. One-stop service for antenatal syphilis screening and prevention of congenital syphilis in Ulaanbaatar, Mongolia: a cluster randomized trial. Sex Transm Dis 2009; 36:714-20.

28. Soeiro CMO, Miranda AE, Saraceni V, Santos MC, Talhari S, Ferreira LCL. Syphilis in pregnancy and congenital syphilis in Amazonas State, Brazil: an evaluation using database linkage. Cad Saúde Pública 2014; 30:715-23.

29. Cunha ACB, Pereira Junior JP, Caldeira CLV, Carneiro VMSP. Diagnóstico de malformações congênitas: impactos sobre a saúde mental de gestantes. Estud Psicol (Campinas) 2016; 33:601-11.

30. Cavalcante EGF, Miranda MCC, Carvalho AZFHT, Lima ICV, Galvão MTG. Partner notification for sexually transmitted infections and perception of notified partners. Rev Esc Enferm USP 2016; 50:448-55.

31. European Centre for Disease Prevention and Control. Public health benefits of partner notification for sexually transmitted infections and HIV. Stockholm: European Centre for Disease Prevention and Control; 2013.

32. Lago E, Rodrigues L, Fiori R, Stein AT. Congenital syphilis: identification of two distinct profiles of maternal characteristics associated with risk. Sex Transm Dis 2004; 31:33-7.

33. Su JR, Brooks LC, Davis DW, Torrone EA, Weinstock HS, Kamb ML. Congenital syphilis: trends in mortality and morbidity in the United States, 1999 through 2013. Am J Obstet Gynecol 2016; 214: 381.e1-9.

34. DiOrio D, Kroeger K, Ross A. Social vulnerability in congenital syphilis case mothers: qualitative assessment of cases in Indiana, 2014 to 2016. Sex Transm Dis 2018; 45:447-51.

35. Bradley E, Taylor L. The American health care paradox: why spending more is getting us less. New York: Public Affairs; 2013. 
36. Santos GS, Oliveira LX, Guimarães AMD, Nardello DM, Braz JM, Barreto IDC. Aspectos epidemiológicos da sífilis congênita associados à escolaridade materna. Rev Enferm UFPE 2016; 10:2845-52.

37. Garbin AJI, Martins RJ, Belila NM, Exaltação SM, Garbin CAS. Reemerging diseases in Brazil: sociodemographic and epidemiological characteristics of syphilis and its under-reporting. Rev Soc Bras Med Trop 2019; 52:e20180226.

38. Lima MG, Santos RFR, Barbosa GJA, Ribeiro GS. Incidência e fatores de risco para sífilis congênita em Belo Horizonte, Minas Gerais, 2001-2008. Ciênc Saúde Colet 2013; 18:499. 506.

39. Lafeta KRG, Martelli Junior H, Silveira MF, Paranaíba LMR. Sífilis materna e congênita, subnotificação e difícil controle. Rev Bras Epidemiol 2016; 19:63-4.

40. Guanabara MO, Araújo MAL, Matsue RY, Barros VL, Oliveira FA. Access of pregnant women to technologies for the prevention and control of congenital syphilis in Fortaleza-Ceará, Brazil. Rev Salud Pública (Bogotá) 2017; 19:73-8.

41. Macêdo VC, Lira PIC, Frias PG, Romaguera LMD, Caires SFF, Ximenes RAA. Fatores de risco para sífilis em mulheres: estudo casocontrole. Rev Saúde Pública 2017; 51:78.

42. Lima MG, Santos RFR, Barbosa GJA, Ribeiro GS. Incidência e fatores de risco para sífilis congênita em Belo Horizonte, Minas Gerais, 2001-2008. Ciênc Saúde Colet 2013; 18:499506.
43. Freitas CHSM, Forte FDS, Galvão MHR, Coelho AA, Roncalli AG, Dias SMF. Inequalities in access to HIV and syphilis tests in prenatal care in Brazil. Cad Saúde Pública 2019; 35:e00170918.

44. Benzaken AS, Sabidó M, Brito I, Bermúdez XPD, Benzaken NS, Galbán E, et al. HIV and syphilis in the context of community vulnerability among indigenous people in the Brazilian Amazon. Int J Equity Health 2017; 16:92.

45. Ormaeche M, Whittembury A, Pun M, SuárezOgnio L. Hepatitis B virus, syphilis, and HIV seroprevalence in pregnant women and their male partners from six indigenous populations of the Peruvian Amazon Basin, 2007-2008. Int J Infect Dis 2012; 16:e724-30.

46. Díaz-Olavarrieta C, Wilson KS, García SG, Revollo R, Richmond K, Paz F, et al. The cooccurrence of intimate partner violence and syphilis among pregnant women in Bolivia. J Womens Health 2009; 18:2077-86.

47. Domingues RMSM, Szwarcwald CL, Souza Junior PRB, Leal MC. Prevalence of syphilis in pregnancy and prenatal syphilis testing in Brazil: birth in Brazil study. Rev Saúde Pública 2014; 48:766-74. 


\section{Abstract}

Brazil has reported an increase in the incidence of both gestational and congenital syphilis, posing a serious public health problem in the country. The study aimed to analyze the relationship between the supply of syphilis diagnosis and treatment in primary care and the incidence rates of gestational and congenital syphilis. An ecological study analyzed these incidence rates and the coverage of diagnostic and therapeutic measures in primary care. The study sample consisted of municipalities (counties) with population over 20,000, with coverage by primary care over 50\%, and where the majority of the teams were assessed in the second cycle of the National Program for Improvement of Access and Quality of Basic Care. Analysis of the effectiveness of detection and treatment measures was based on the development of the Index of Variation in Vertical Syphilis Transmission. The administration of penicillin and application of the rapid test in these municipalities showed median rates of $41.9 \%$ and $67.14 \%$, respectively, with regional differences. Median incidence of gestational syphilis was 6.24 (IQR: 2.63-10.99) in municipalities with a higher supply of the rapid test and 3.82 (IQR: 0.00-8.21) in those with a lower supply, indicating an increase in detection capacity. Municipalities with a reduction in vertical transmission showed higher median rates of teams with supply of rapid testing and (83.33\%; IQR: 50.00100.00) and administration of penicillin (50.00\%; $I Q R: 11.10-87.50)$, demonstrating a relationship between these measures and the reduction in congenital syphilis. The findings indicate the need to expand these services and to reinforce the importance of reducing vertical transmission.

Congenital Syphilis; Maternal and Child Health; Primary Health Care; Incidence; Health Evaluation

\section{Resumen}

Brasil ha registrado un aumento en las incidencias de sifilis gestacional y congénita, revelándose como un importante problema de salud pública en Brasil. El estudio tuvo como objetivo analizar la relación entre las ofertas de diagnóstico y el tratamiento de la sífilis en la atención básica, así como las incidencias de sifilis gestacional y congénita. Se realizó un estudio ecológico, analizando las incidencias de esos problemas de salud y la cobertura de las acciones diagnósticas y terapéuticas en la atención básica. La muestra del estudio estuvo compuesta por municipios con una población por encima de los 20.000 habitantes, con una cobertura de atención básica superior a un 50\%, y en los que la mayoría de los equipos se evaluó en el segundo ciclo del Programa Nacional de Mejora de Acceso y Calidad de la Atención Básica Para analizar la efectividad de las acciones de detección y tratamiento se desarrolló el Índice de Variación de la Transmisión Vertical de Sífilis. La administración de la penicilina y la realización del test rápido en estos municipios obtuvieron medias iguales a $41,9 \%$ y $67,14 \%$, respectivamente, con diferencias regionales. La media de la incidencia de sifilis gestacional fue 6,24 (IIQ: 2,63-10,99) en municipios con mayor oferta de test rápido y de 3,82 (IIQ: $0,00-8,21)$ en aquellos con oferta inferior, apuntando un aumento en la capacidad de detección. Los municipios con una reducción de la transmisión vertical presentaban mayores medias de los porcentajes de equipos con oferta de tests rápidos (83,33\%; IIQ: 50,00-100,00) y administración de penicilina (50,00\%; IIQ: 11,10-87,50), demostrando la relación entre estas acciones y la reducción de sífilis congénita. Los resultados indican la necesidad de que exista una ampliación de esta oferta de servicios y refuerza la importancia en la reducción de la transmisión vertical.

Sífilis Congénita; Salud Materno-Infantil; Atención Primaria de Salud; Incidencia; Evaluación en Salud
Recebido em 20/Abr/2019

Versão final reapresentada em 20/Ago/2019 Aprovado em 03/Set/2019 